\title{
Surface Dynamics and Phononic Properties of 2D Field Tunable Auxetic Crystal
}

\author{
D. TwaróG ${ }^{a, *}$, D. TrzupeK ${ }^{a, b}$ AND P. Zieliński ${ }^{a, c}$ \\ ${ }^{a}$ The H. Niewodniczański Institute of Nuclear Physics, PAS \\ Radzikowskiego 152, 31-342 Kraków, Poland \\ ${ }^{b}$ Institute of Physics, Jagiellonian University \\ Reymonta 4, 30-059 Kraków, Poland \\ ${ }^{c}$ Institute of Physics, Cracow Technical University \\ Podchorążych 1, 30-084 Kraków, Poland
}

\begin{abstract}
The lattice in-plane dynamics is studied as a function of external field in a model of auxetic crystal consisting of rigid polar rods and of elastic springs. The phase speed of the transverse acoustic wave propagating parallel to the polar rods is shown to be higher than the speed of the longitudinal wave at strong fields. An absolute stop band for the lattice waves opens in the whole Brillouin zone with increasing field. The surface waves and resonances at a surface parallel to the rods are studied. Infinitely narrow resonances called exceptional surface waves are found within bulk bands at certain specific values of the parameters characterizing the surface. Generally, the surface layer should be significantly heavier and stiffer than the substrate for the phenomenon to occur.
\end{abstract}

PACS numbers: 63.20.D-, 68.35.Ja, 62.20.dj

\section{The model}

The geometry of the model stems from the generic structure proposed by Evans [1] for auxetic materials. The model consists of rigid rods connected by elastic springs as depicted in Fig. 1. The springs $\alpha, \beta$, and $\gamma$ are supposed to take their free lengths in the equilibrium state in contrast with stretched springs of earlier models [2]. The dynamical properties of the model can be modified or even tuned by an external field if the rods possess dipole moments. In the present study we assume a field applied in the horizontal direction that is along the axis $x_{1}$ of Fig. 1. The existence of such a field manifests itself by an additional torque restoring the horizontal orientation of the rods. The corresponding rotational force constant is called $K$. This is to some extent analogous to the rotational background potential of Ref. [3]. In what follows we study the bulk and the surface properties of the model as functions of the intensity of the external field, i.e. of the torque force constant $K$.

The structure shows a base centered symmetry $C 2 \mathrm{~mm}$ with one rod per the primitive unit cell. Consequently, the rectangular unit cell of edges $a$ and $b=a \sqrt{3}$ contains two rods. Every rod has three degrees of freedom $\left(u_{1}, u_{2}, \phi\right)$ where the vector components $\left(u_{1}, u_{2}\right)$ describe the displacement of the rod's mass center from its equilibrium position and the angle $\phi$ is the inclination of the rod with respect to the horizontal orientation. Consequently,

* corresponding author; e-mail: Dariusz.Twarog@ifj.edu.pl

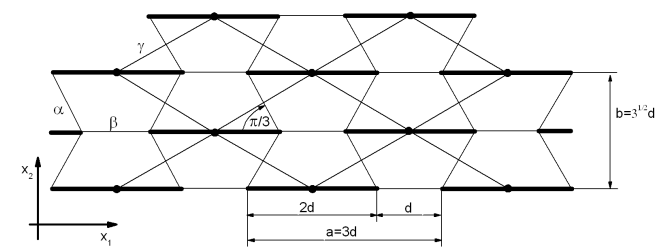

Fig. 1. Geometry of model. Rods are represented by thick lines and force constants by thin lines labeled in Greek. Distance between neighboring chains is $b / 2$ and between centers of two rods in the same chain is $a(d=1)$.

written in the frequency and wave vector representation, the equations of motion are given by a system of homogeneous linear equations for $\left(u_{1}, u_{2}, \phi\right)$ with the following $3 \times 3$ dynamical matrix $\boldsymbol{M}$ :

$$
\begin{aligned}
M_{11} & =m \omega^{2}+2 \beta[\cos (k, a)-1] \\
+(\alpha+3 \gamma)\left[\cos \left(\frac{1}{2} k_{2} b\right) \cos \left(\frac{1}{2} k_{2} b\right)-1\right], & \\
M_{12} & =\sqrt{3}(\alpha-\gamma) \sin \left(\frac{1}{2} k_{1} a\right) \sin \left(\frac{1}{2} k_{2} b\right), \\
M_{13} & =\mathrm{i} \sqrt{3} \alpha \cos \left(\frac{1}{2} k_{1} a\right) \sin \left(\frac{1}{2} k_{2} b\right), \\
M_{21} & =\sqrt{3}(\alpha-\gamma) \sin \left(\frac{1}{2} k_{1} a\right) \sin \left(\frac{1}{2} k_{2} b\right),
\end{aligned}
$$




$$
\begin{aligned}
& M_{22}=m \omega^{2} \\
& +(3 \alpha+\gamma)\left[\cos \left(\frac{1}{2} k_{1} a\right) \cos \left(\frac{1}{2} k_{2} b\right)-1\right], \\
& M_{23}=-3 \mathrm{i} \alpha \sin \left(\frac{1}{2} k_{1} a\right) \cos \left(\frac{1}{2} k_{2} b\right), \\
& M_{31}=-\mathrm{i} \sqrt{3} \alpha \cos \left(\frac{1}{2} k_{1} a\right) \sin \left(\frac{1}{2} k_{2} b\right), \\
& M_{32}=3 \mathrm{i} \alpha \sin \left(\frac{1}{2} k_{1} a\right) \cos \left(\frac{1}{2} k_{2} b\right) \\
& M_{33}=\frac{J \omega^{2}}{d}-3 \alpha\left[1+\cos \left(\frac{1}{2} k_{1} a\right) \cos \left(\frac{1}{2} k_{2} b\right)\right] \\
& -K
\end{aligned}
$$

where $m$ is the mass and $J$ is the moment of inertia of the rods.

The condition for vanishing of the determinant of the matrix $\operatorname{det}(\boldsymbol{M})=0$ gives the dispersion curves of the model crystal. There are three eigenfrequencies at any wave vector $\left(k_{1}, k_{2}\right)$. Two of them tend linearly to zero at small wave vectors. This part of the spectrum constitutes a long wavelength or acoustic limit.

\section{Long wavelength limit}

To describe the acoustic part of the lattice waves spectrum i.e. for $|\boldsymbol{k}| \ll 1 / a$ and $\omega \propto k$, we expand the potential energy per unit cell in terms of the components of the gradient of the displacement vector

$$
\eta_{i j}=\frac{\partial u_{i}}{\partial x_{j}} .
$$

The energy of the system is a quadratic form of the quantities $\eta_{i j}$ :

$$
E_{\mathrm{el}}=\frac{1}{2} \sum_{i j, k l} \eta_{i j} \tilde{C}_{i j k l} \eta_{k l},
$$

where the coefficients $\tilde{C}_{i j k l}$ are analogs of the elastic constants. Because of the lack of the rotational invariance due to the external field $K$ the only symmetry that is shown by the coefficients $\tilde{C}_{i j k l}$ is $\tilde{C}_{i j k l}=\tilde{C}_{k l i j}$, whereas the interchange within each pair of the indices $i j$ and $k l$ does not leave the coefficients invariant. Expressed by the force constants the coefficients of the acoustic energy of Eq. (2.2) read

$$
\begin{aligned}
& \tilde{C}_{1111}=\frac{\sqrt{3}}{4} \alpha+2 \sqrt{3} \beta+\frac{3 \sqrt{3}}{4} \gamma, \\
& \tilde{C}_{2222}=\frac{\sqrt{3}}{4} \alpha+\frac{\sqrt{3}}{12} \gamma, \\
& \tilde{C}_{1122}=\tilde{C}_{2211}=\frac{\sqrt{3}}{4}(\gamma-\alpha), \\
& \tilde{C}_{2121}=\frac{\sqrt{3}}{4} \frac{K(3 \alpha+\gamma)+6 \alpha \gamma}{K+6 \alpha},
\end{aligned}
$$

$$
\begin{aligned}
& \tilde{C}_{1212}=\frac{\sqrt{3}}{12} \frac{K(3 \gamma+\alpha)+18 \alpha \gamma}{K+6 \alpha}, \\
& \tilde{C}_{1221}=\tilde{C}_{2112}=\frac{3 \sqrt{3} \alpha^{2}}{2(6 \alpha+K)}+\frac{\sqrt{3}}{4}(\gamma-\alpha) .
\end{aligned}
$$

One can easily check that at $K \rightarrow 0$, the usual symmetry of the elastic constants $\tilde{C}_{i j k l}=\tilde{C}_{j i k l}=\tilde{C}_{i j l k}$ is restored. As expected from symmetry the present parameters $\tilde{C}_{i j k l}$ for $i=j, k=l$ are independent of the torque force constant $K$. The phase speeds of the longitudinal waves $c_{\mathrm{L}}^{(1,0)}=\left(\tilde{C}_{1111} / \rho\right)^{1 / 2}$ and $c_{\mathrm{L}}^{(0,1)}=\left(\tilde{C}_{2222} / \rho\right)^{1 / 2}$ in the horizontal and vertical directions, respectively, are also independent of the torque force constant $K$. The phase speeds of the transverse waves $c_{\mathrm{T}}^{(1,0)}=\left(\tilde{C}_{2121} / \rho\right)^{1 / 2}$ and $c_{\mathrm{T}}^{(0,1)}=\left(\tilde{C}_{1212} / \rho\right)^{1 / 2}$ depend explicitly on the constant $K$. In fact, the transverse waves produce simple but not pure shears [4]. The Poisson ratio in the $(1,0)$ and $(0,1)$ directions are given by $\tilde{C}_{1122} / \tilde{C}_{2222}$ and $\tilde{C}_{1122} / \tilde{C}_{1111}$, respectively, and are independent of the torque force constant $K$.

\section{Reversal of transverse and longitudinal wave speeds with external field}

Elastic parameters $\tilde{C}_{1212}$ and $\tilde{C}_{2121}$ (Eqs. (2.6) and (2.8)) are functions of the torque force constant $K$. An insight into Eqs. (2.3) and (2.6) shows that the parameter $\tilde{C}_{2121}$ becomes larger than $\tilde{C}_{1111}$ if the torque force constant $K$ exceeds a certain value, i.e. $K>$ $3 \alpha(\alpha+8 \beta+2 \gamma)(\alpha-4 \beta-\gamma)^{-1}$. Then the phase speed of the transverse wave propagating in the direction $(1,0)$ becomes larger than the phase speed of the longitudinal wave in the same direction. Analogous phenomenon takes place in direction $(0,1)$ perpendicular to the surface at $K>3 \alpha(3 \alpha-2 \gamma)(\gamma-\alpha)^{-1}$ which corresponds to $\tilde{C}_{1212}>C_{2222}$. In fact, most of materials free of external fields or loads usually show the phase speeds of longitudinal waves larger than the speeds of the transverse waves in the same direction. Therefore, the occurrence of the reversal of the wave speeds described above is worth further studies. The phenomenon may also have practical applications.

\section{Field induced phononic properties}

The effect of the torque force constant $K$ onto the dispersion relations in the present models is illustrated by dispersion relations calculated along the path $\Gamma X Y \Gamma$ in the Brillouin zone. Figures 2-4 show the dispersion relations with parameters corresponding to an acoustically isotropic case, i.e. for $\tilde{C}_{1111}=\tilde{C}_{2222}$ and $2 \tilde{C}_{1221}=$ $\tilde{C}_{1111}-\tilde{C}_{1122}$ in the limit $K \rightarrow 0$. This corresponds to $\beta=-\gamma / 3$ and $\alpha=4 \gamma / 3$. We have chosen arbitrarily $\gamma=10^{4}$. As it is seen in Fig. 4a an absolute stop band (the Bragg gap) opens throughout the whole Brillouin zone. This means that no lattice wave can propagate in a certain range of frequencies. The wave speeds of 

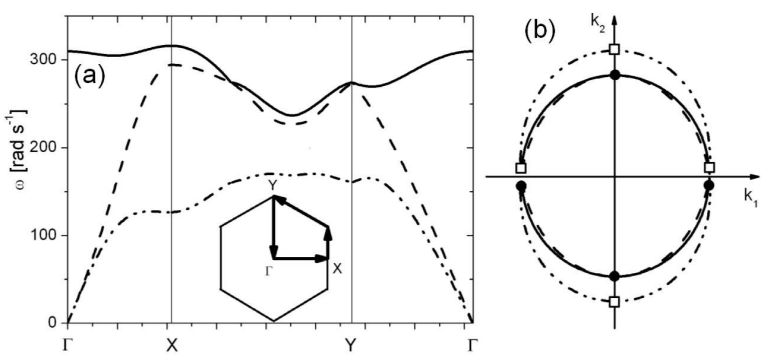

Fig. 2. Dispersion curves along path given in inset (a) and slowness surfaces (constant $\omega$ surfaces at small wave vectors) for acoustic waves at $K=1.6 \times 10^{4}$ (b). Black dots indicate longitudinal and open squares - transverse waves. Solid reference circle corresponds to $K=0$.

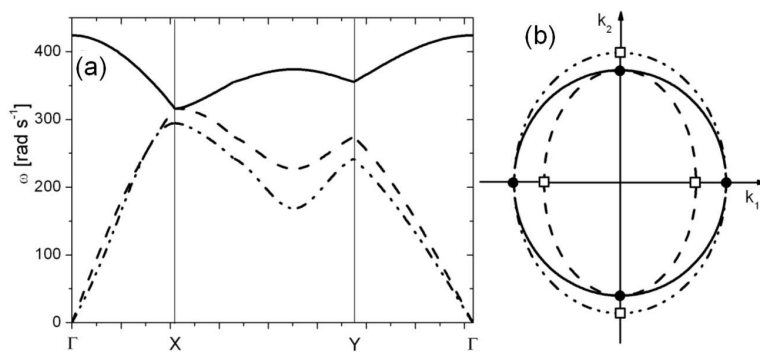

Fig. 3. The same as in Fig. 2, but with $K=10^{5}$. Opening of absolute stop band.

acoustic waves are illustrated in Figs. 2b, 3b and $4 \mathrm{~b}$ by surfaces of constant frequency traced in the reciprocal space. The slower wave then corresponds to the outer surface and the faster wave to the inner surface. Such surfaces (which reduce to contours in two dimensions) are called slowness surfaces [5]. An interesting phenomenon occurring in our model is that the slower wave in one direction is longitudinal and the transverse one is slower in the perpendicular direction although both waves belong to one common slowness surface.

\section{Exceptional surface wave}

A model of surface is obtained by removal of the half plane $x_{2}>0$ in Fig. 1. The mass, moment of inertia, stretching force constant and torque force constant: $m_{\mathrm{S}}$, $I_{\mathrm{S}}, \beta_{\mathrm{S}}, K_{\mathrm{S}}$, of the topmost chain of rods may differ from those in the bulk. The bulk bands and dispersion relations of the surface waves for the following values of the bulk parameters: $m=1, J=0, \alpha=10^{4}, \gamma=3 \alpha / 4, \beta=$ $-\gamma / 3$ (auxetic case), $K=K_{\mathrm{S}}=10^{3}\left(c_{\mathrm{T}}^{(1,0)}<c_{\mathrm{L}}^{(1,0)}\right)$ and of the surface parameters: $m_{\mathrm{S}}=127.09, \beta_{\mathrm{S}}=50886.27$ and $J_{\mathrm{S}}=0$ are depicted in Fig. 5a. The choice of parameters corresponds to a particularity of this model, namely the existence of an infinitely narrow resonance within a bulk band. The resonance is called here exceptional surface wave (ESW). The imaginary part of the resonance frequency, which amounts to the width of the resonance, is shown in Fig. 5b. It is remarkable that at

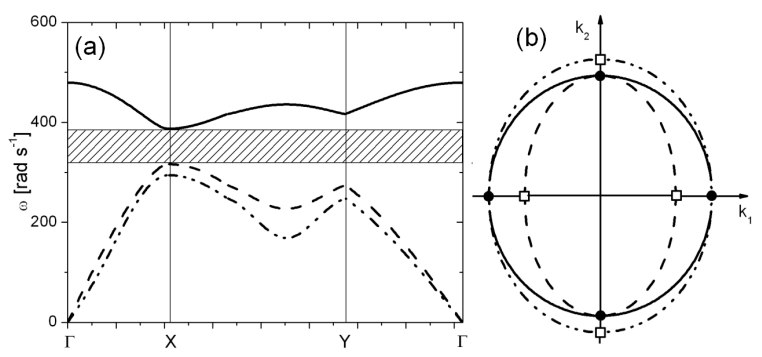

Fig. 4. The same as in Fig. 2, but with $K=1.5 \times 10^{5}$. Absolute stop band gap (shaded) opened in the whole Brillouin zone.

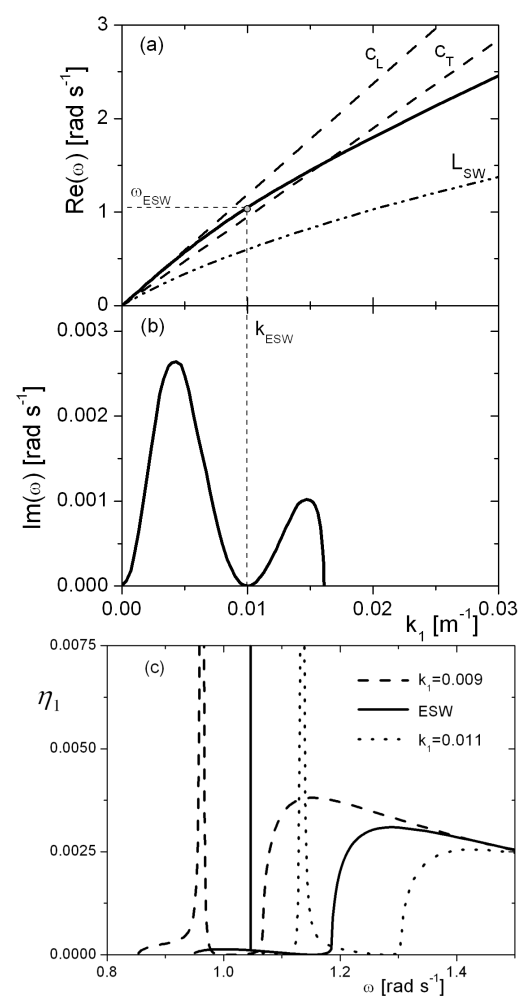

Fig. 5. Boundaries of bulk bands (dashed lines), surface resonance dispersion curve (solid line) and longitudinal surface wave $L_{\mathrm{SW}}$ (dash-dot-dotted line) (a). Imaginary part of resonance frequency (b) and local density of states close to ESW (c).

certain value of wave vector the imaginary part of frequency becomes zero which corresponds to the infinite lifetime of the resonance. The local density of states as a function of frequency is visible in Fig. 5c for the wave vector $k_{1}=0.01 / d=0.03 / a$ corresponding to the exceptional surface wave and to two close wave vectors.

\section{Conclusions}

The present model offers a possibility of tuning some useful dynamical properties of an auxetic crystal by an external field. If the field is sufficiently strong, a com- 
plete stop band opens throughout whole Brillouin zone. The width of the Bragg gap is strictly dependent on $K$ which may be useful in fabrication of new generations of acoustic barriers tunable by external field. This should be compared with other ways to generate phononic properties: geometrical [6] and tunable by other type of parameters [7]. The present model confirms the possibility of existence of an infinitely narrow surface resonances (exceptional surface waves) within bulk bands formerly observed in a continuous medium model [8]. The condition for it to occur is a sufficiently high mass of the surface rods and sufficiently strong interactions within the surface layer.

\section{Acknowledgments}

This work is part of project "Ion and plasma nanotechnologies for materials formed on the basis of C and Si" in the frame of operational programme Innovative Economy (Poland). The National Network MANAR of the Ministry of Science and Higher Education has provided a significant support. The contribution of the coopera- tion project of the Polish Academy of Sciences and Centre National de la Recherche Scientifique (France) is acknowledged.

\section{References}

[1] K.E. Evans, M.A. Nkansah, I.J. Hutchinson, S.C. Rogers, Nature 353, 124 (1991).

[2] A. Sparavigna, Phys. Rev. B 76, 134302 (2007).

[3] A.A. Vasiliev, S.V. Dimitriev, Y. Ishibashi, T. Shigenari, Phys. Rev. B 65, 094101 (2002).

[4] J.F. Nye, Physical Properties of Crystals, 1st ed., Clarendon, Oxford 1957, p. 104.

[5] T. Paszkiewicz, M. Pruchnik, P. Zieliński, Eur. Phys. J. B 24, 327 (2001).

[6] H. Larabi, Y. Pennec, B. Djafari-Rouhani, J.O. Vasseur, Phys. Rev. E 75, 066601 (2007).

[7] F. Liu, F. Cai, Y. Ding, Z. Liu, Appl. Phys. Lett. 92, 103504 (2008).

[8] D. Twaróg, P. Zieliński, Phase Transit. 79, 577 (2006). 